\title{
The therapeutic potential of attentional bias modification training for insomnia: study protocol for a randomised controlled trial
}

Umair Akram $^{1 *}$ (1), Bronwyn Milkins², Antonia Ypsilanti ${ }^{1}$, John Reidy ${ }^{1}$, Lambros Lazuras $^{1}$, Jodie Stevenson ${ }^{1,3}$, Lies Notebaert ${ }^{2}$ and Nicola L. Barclay ${ }^{4}$

\begin{abstract}
Background: The efficacy of attentional bias modification (ABM) as a treatment for anxiety and depression has been extensively studied with promising results. Despite some evidence of sleep-related attentional biases in insomnia, only a small number of studies, yielding mixed results, have examined the application of ABM in insomnia. This study specifically aims to determine whether ABM can reduce (i) the presence of an attentional bias for sleep-related threatening words; (ii) insomnia symptom severity; (iii) sleep onset latency; and (iv) pre-sleep cognitive arousal amongst individuals with insomnia compared to a non-treatment control group of individuals with insomnia.

Methods/design: We propose a randomised controlled trial of 90 individuals from the general population who meet the criteria for Insomnia Disorder. Following an initial examination for the presence of a sleep-related attentional bias using the dot-probe paradigm, participants will be randomised to an online attentional bias modification training condition, or to a standard attentional bias task (non-treatment) control condition. Both conditions will be delivered online by a web platform. All participants allocated to the non-treatment control group will be offered ABM training once the study is complete. The primary outcome will be the attentional bias indices of vigilance and disengagement and self-reported insomnia symptoms, sleep onset latency and pre-sleep cognitive arousal. Attentional bias and insomnia symptoms will be assessed at baseline (day 1) and post-treatment (2 days after the final training session: day 9). Insomnia symptoms will be again assessed at follow-up (day 16). Secondary outcomes include examining whether sleep associated monitoring and worry are related to a sleep-related attentional bias in insomnia, and whether such reports reduce following ABM. All main analyses will be carried out on completion of follow-up assessments. The trial is supported by the Department of Psychology, Sociology and Politics at Sheffield Hallam University.
\end{abstract}

Discussion: This study will extend the research base examining the efficacy of attentional bias modification for insomnia.

Trial registration: ISRCTN (ISRCTN11643569, registered on 5 June 2018).

Keywords: Insomnia, Attentional bias, Attention bias modification, ABM, Cognitive bias, Randomised controlled trial

\footnotetext{
* Correspondence: u.akram@shu.ac.uk

1 Department of Psychology, Sociology and Politics, Sheffield Hallam

University, Collegiate Crescent, Sheffield, South Yorkshire S10 2BP, UK

Full list of author information is available at the end of the article
}

(c) The Author(s). 2018 Open Access This article is distributed under the terms of the Creative Commons Attribution 4.0 International License (http://creativecommons.org/licenses/by/4.0/), which permits unrestricted use, distribution, and reproduction in any medium, provided you give appropriate credit to the original author(s) and the source, provide a link to the Creative Commons license, and indicate if changes were made. The Creative Commons Public Domain Dedication waiver (http://creativecommons.org/publicdomain/zero/1.0/) applies to the data made available in this article, unless otherwise stated. 


\section{Background}

Cognitive models of insomnia propose that selective attention for sleep-related information acts as a maintaining factor of the disorder $[1,2]$. Such attention may be placed on internal (i.e. bodily sensations) or external (i.e. outside noises) cues and may occur on awakening, during the day and in the pre-sleep period. It is theorized that selective attention for sleep-related information contributes to increased physiological and cognitive arousal, and excessive worry concerning the negative daytime consequences (i.e. impaired daytime functioning) which are perceived to precede night-time sleep difficulty. These processes are said to generate and maintain sleep-specific anxiety, which ultimately produces a state that is incompatible with the initiation of sleep. In support of this notion, several studies have investigated attentional biases to sleep-related cues in insomnia using experimental reaction time (i.e. dot-probe, flicker, and emotional Stroop) and eye-tracking paradigms [3-12]. The majority of these studies suggest that poor sleepers and individuals with insomnia display attentional biases towards sleep-related words and images compared with normal-sleepers [3-5, 7-13].

In disorders such as anxiety and depression, where attentional biases are prominent, it has been evidenced that attention biases for negative, disorder salient, information can be alleviated using attentional bias modification (ABM) tasks [14]. In these studies, attentional avoidance of negative information is encouraged through use of a modified dot-probe task where probes always appear in the location opposite negative stimuli. Over time, this procedure has been known to 'train' an individual's attention away from negative information related to their specific condition [14]. When applied prior to an event perceived as threatening to a specific population (i.e. anxious individuals asked to speak in public), the immediate effects of ABM appear to be most prominent [15].

Recent research has examined the efficacy of delivering $\mathrm{ABM}$ immediately prior to bed as a treatment for individuals reporting elevated disposition to experiencing poor sleep quality $[16,17]$. Specifically, these studies obtained evidence that individuals provided with ABM also reported improved subjective sleep quality, reduced pre-sleep arousal, and reduced sleep onset latency relative to control conditions where a standard attentional bias task was delivered. However, these studies utilised a measure of disposition to experiencing poor sleep to recruit participants, rather than a measure of disposition to experiencing insomnia-specific symptoms, which limits the generalisability of these studies. To that end, Lancee et al. [18] explored the therapeutic effect of ABM amongst those meeting diagnostic criteria for insomnia. Whilst no therapeutic effects were observed in this study, ABM was administered in the evening between 7 and $11 \mathrm{pm}$, rather than immediately prior to bed when biased attention may be most prominent [16].

The present research aims to further examine the therapeutic potential of attentional bias modification for the treatment of insomnia-specific symptoms. Previous research has proved to be efficacious where delivery of $\mathrm{ABM}$ and control tasks are alternated amongst the same sample [16]. Whilst this research highlights promising outcomes for those experiencing sleep disturbance, these outcomes cannot be extrapolated to individuals with insomnia. Moreover, the within-subjects alternating nature of this research may also prevent any potential accumulation of improved sleep from being otherwise observed. Therefore, the current study will implement a between-subjects design using a sample of individuals meeting the diagnostic criteria, who will be randomly allocated to either a treatment or non-treatment control condition. This will allow for observation of ABM over repeated nights and for the presence of any potential $\mathrm{cu}$ mulative changes in outcome measures. Specifically, using a similar modified dot-probe task to Milkins et al. [16], we aim to determine whether ABM can reduce (i) the presence of an attentional bias for sleep-related negative words characterised by either vigilance (i.e. speeding of attention towards salient stimuli) or disengagement (i.e. difficulty in shifting attention away from salient stimuli); (ii) insomnia symptom severity; (iii) sleep onset latency; and (iv) pre-sleep cognitive arousal amongst individuals with insomnia compared to a non-treatment control group of individuals with insomnia. As a secondary aim, we also seek to determine whether initial attentional bias is related to greater self-reports of sleep-associated monitoring behaviour and sleep-related worry, and whether such reports reduce following ABM.

\section{Methods/design \\ Trial design}

This study is a double-blind randomised controlled trial examining the efficacy of ABM for insomnia delivered over the internet (see Fig. 1. for summary). The study has received ethical approval from the Sheffield Hallam University Research Ethics Committee (reference number: ER5451619).

\section{Participants and recruitment}

We will recruit a total of 90 adult members from the general population who meet the Diagnostic and Statistical Manual of Mental Disorders, $5^{\text {th }}$ edition (DSM-5) criteria for Insomnia Disorder [19]. Potential participants will be recruited through the website 'call for participants' and various insomnia treatment forums and will be assessed online for eligibility. Those interested in taking part will complete the Insomnia Severity Index 


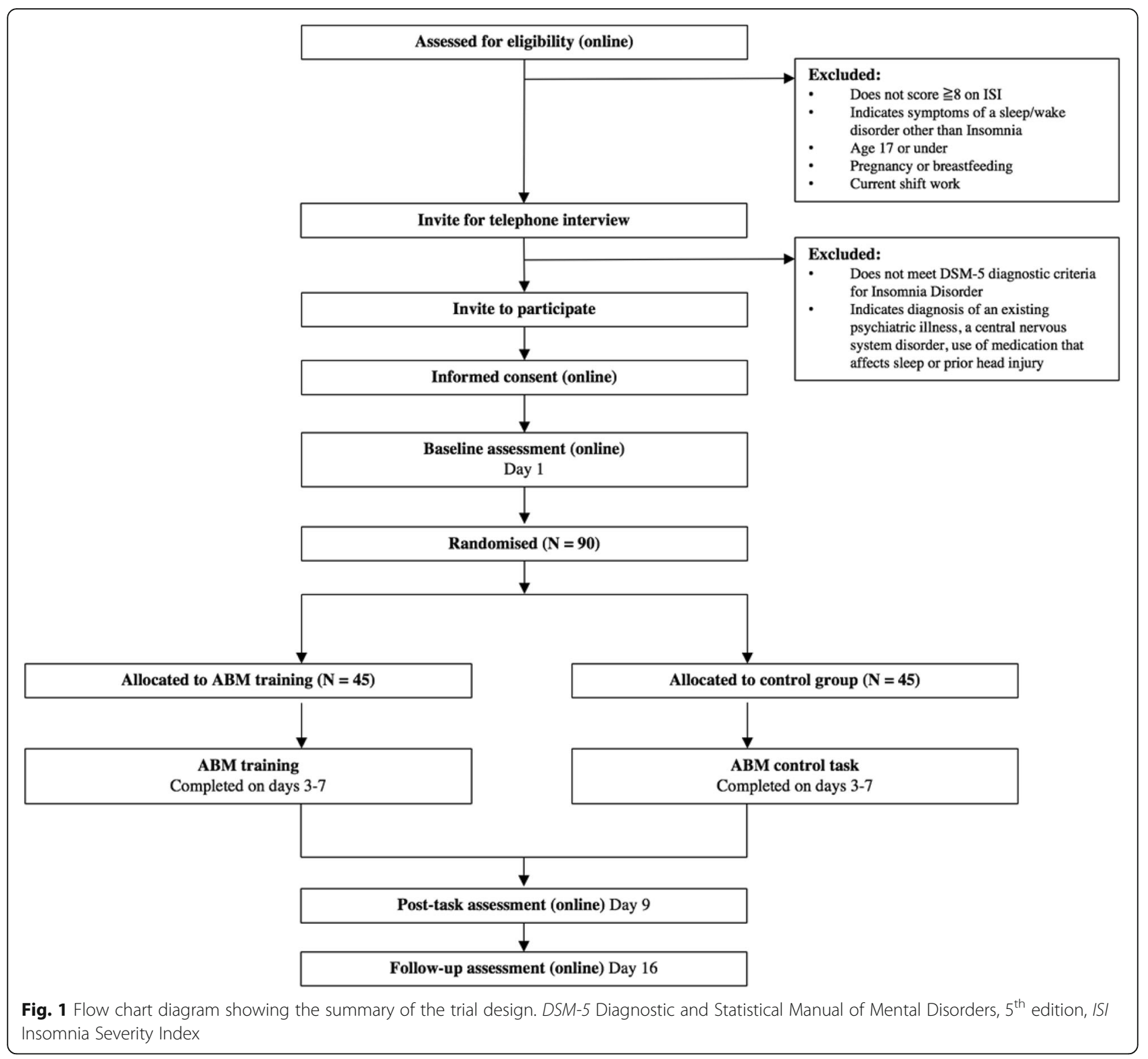

(ISI: [20]), and SLEEP-50 [21] measures (see 'Questionnaire Measures' section for more detail). Those indicating subclinical insomnia using the ISI, and do not indicate the presence of another sleep/wake disorder other than insomnia on the SLEEP-50 will be invited for a telephone interview to determine the presence of DSM-5 Insomnia Disorder. Telephone interviews will be conducted by the principal investigator (UA).

Specifically, individuals with insomnia must report dissatisfaction with sleep characterized by either a difficulty initiating or maintaining sleep or early morning awakenings. Insomnia should be present for 3 or more nights per week, for at least 3 months, and cause significant daytime impairment. Finally, these conditions must be met despite an adequate opportunity to sleep. Those who report symptoms of a sleep/wake disorder other than insomnia, an existing psychiatric illness, a central nervous system disorder, use of medication that may affect sleep, a prior head injury, pregnancy or breast-feeding or current shift-work will be ineligible to take part. Those meeting the criteria for Insomnia Disorder will be invited to participate and will be provided with an email link for the online study. All participants must have normal to corrected vision.

\section{Randomisation and allocation concealment}

This study will use a simple randomisation with an allocation ratio of $1: 1$ which will be carried out by the automated online system. As such, the research team will 
not be able to influence randomisation and will not have access to future allocations.

\section{Blinding}

This will be a double-blind trial. Once invited to participate, all assessments and group allocation will occur online, and the research team will be blind to outcomes during the trial. Participants will not be aware whether they are in the experimental or control condition. It is relevant to note that the research team is unlikely to have any contact with the participants, and as such it will be unlikely that they are able to bias allocation or assessment.

\section{Word pairs}

\section{Assessment pairs}

In this study we will use the same set of 48 sleep-negative and neutral word pairs as used by Milkins et al., ([16]; see Tables 1 and 2 for word pairs). A subset

Table 1 List of sleep-negative/neutral and neutral-neutral word pairs for attentional bias assessment points (baseline and post-treatment)

\begin{tabular}{|c|c|c|c|}
\hline \multicolumn{2}{|c|}{ Sleep-negative and neutral pairs } & \multicolumn{2}{|c|}{ Neutral-neutral pairs } \\
\hline $\begin{array}{l}\text { Sleep-negative } \\
\text { word }\end{array}$ & $\begin{array}{l}\text { Matched } \\
\text { neutral word }\end{array}$ & $\begin{array}{l}\text { Neutral } \\
\text { word }\end{array}$ & $\begin{array}{l}\text { Matched } \\
\text { neutral word }\end{array}$ \\
\hline Ache & Melt & Graft & Males \\
\hline Lethargy & Teaspoon & Featuring & Scrubbing \\
\hline Agitated & Carriers & Cushion & Specify \\
\hline Naps & Kits & Meals & Chord \\
\hline Angry & Curve & Led & Lot \\
\hline Overactive & Clearances & Stairs & Mirror \\
\hline Coffee & Beside & Invoice & Prodigy \\
\hline Sleepy & Wallet & Playful & Tramped \\
\hline Delirious & Fragments & Margin & Pencil \\
\hline Snoring & Penguin & Tops & Laps \\
\hline Disastrous & Curriculum & Book & News \\
\hline Tense & Trick & Habit & Steel \\
\hline Disorder & Compost & Sandals & Potters \\
\hline Sluggish & Decorate & Attendants & Speculates \\
\hline Distress & Creature & Invents & Drafted \\
\hline Restless & Receiver & Graft & Disarm \\
\hline Exhausted & Developed & Point & Water \\
\hline Snoring & Penguin & Thankful & Entrants \\
\hline Hopeless & Borrowed & Vaccine & Embrace \\
\hline Sleepy & Wallet & Wriggles & Fixation \\
\hline Inadequate & Initiative & Pear & Bath \\
\hline Nightmare & Volunteer & Trait & Stand \\
\hline Irritable & Appliance & Days & Motel \\
\hline Drowsy & Umpire & Eraser & Places \\
\hline
\end{tabular}

Table 2 List of sleep-negative/neutral word pairs for ABM experimental and control conditions

\begin{tabular}{|c|c|c|c|}
\hline \multicolumn{2}{|c|}{ Sleep-negative and neutral pairs } & \multicolumn{2}{|c|}{ Neutral-neutral pairs } \\
\hline $\begin{array}{l}\text { Sleep-negative } \\
\text { word }\end{array}$ & $\begin{array}{l}\text { Matched } \\
\text { neutral word }\end{array}$ & $\begin{array}{l}\text { Sleep-negative } \\
\text { word }\end{array}$ & $\begin{array}{l}\text { Matched } \\
\text { neutral word }\end{array}$ \\
\hline Ache & Melt & Lazy & Hint \\
\hline Afraid & Artist & Lethargy & Teaspoon \\
\hline Agitated & Carriers & Medication & Bottleneck \\
\hline Alarm & Spray & Naps & Kits \\
\hline Angry & Curve & Nightmare & Volunteer \\
\hline Anxiety & Express & Overactive & Clearances \\
\hline Coffee & Beside & Panicky & Infuser \\
\hline Conflict & Advanced & Restless & Receiver \\
\hline Delirious & Fragments & Sad & Cup \\
\hline Desperate & Aesthetic & Sick & Pull \\
\hline Disastrous & Curriculum & Sleepless & Advisable \\
\hline Disengaged & Nonfiction & Sleepy & Wallet \\
\hline Disorder & Compost & Sluggish & Decorate \\
\hline Distract & Brochure & Snoring & Penguin \\
\hline Distress & Creature & Stress & Links \\
\hline Drowsy & Umpire & Suffer & Branch \\
\hline Exhausted & Developed & Tense & Trick \\
\hline Fatigue & Passive & Time & Look \\
\hline Hopeless & Borrowed & Tired & Plain \\
\hline Illness & Mustard & Unpleasant & Expedition \\
\hline Inadequate & Initiative & Wakeful & Trolley \\
\hline Irrational & Flashlight & Work & Life \\
\hline Irritable & Appliance & Workload & Handbags \\
\hline Jittery & Thermos & Worry & Theme \\
\hline
\end{tabular}

$A B M$ attention bias modification

of 24 of these sleep-negative and neutral word pairs will be used alongside 24 neutral-neutral word pairs. Each of the words will be matched on frequency and length.

\section{$A B M$ control and experimental pairs}

Here, we will use the full set of 48 sleep-negative and neutral word pairs as used by Milkins et al., [16].

\section{Questionnaire measures \\ Primary measures}

Insomnia severity Insomnia symptoms will be assessed using the Insomnia Severity Index [20] (ISI). The ISI consists of seven items examining the severity of insomnia symptoms over the past 2 weeks including difficulty initiating and maintaining sleep and awakening too early. Items are scored on a 5-point Likert scale, with total scores ranging from 0 to 28 . Higher scores represent 
greater insomnia severity, with scores of $\geq 8$ indicative of subclinical insomnia.

Consensus sleep diary The critical measure of sleep onset latency (SOL) will be assessed as part of a standardized sleep diary [22], completed by participants each morning of the study. Here, participants will record in minutes the estimated time taken to fall asleep the previous night.

Pre-sleep cognitive arousal To assess pre-sleep cognitive arousal before and after delivery of the ABM/ control tasks, we will use the Pre-Sleep Arousal Scale (PSAS: [23]). The scale contains 16 items related to the kinds of thoughts and sensations experienced in the pre-sleep period which can contribute to sleeplessness.

\section{Secondary measures}

Sleep associated monitoring Monitoring will be assessed using the Sleep Associated Monitoring Index (SAMI: [24]), a 30-item measure comprising of eight subscales: daytime monitoring for body sensations; calculation of time; waking monitoring for body sensations; pre-sleep monitoring for body sensations consistent with falling asleep; daytime monitoring of functioning; pre-sleep monitoring for body sensations inconsistent with falling asleep; pre-sleep monitoring the clock; and pre-sleep monitoring the environment. Items are measured on a 5-point scale on which participants indicate what is true for them over the past week $(1=$ not at all, 5 $=$ all the time). Mean scores for subscales reflect total subscale scores divided by the number of items in the scale.

Anxiety and preoccupation with sleep Anxiety and sleep-related worry will be examined using the Anxiety and Preoccupation about Sleep Questionnaire (ASPQ: [25]) and the Sleep Anticipatory Anxiety Scale (SAAQ: [26]). The ASPQ is comprised of 10 items that examine the extent to which participants have been worried about the nature and consequences of their sleep problem over the past 3 days. The SAAQ consists of 10 items that examine sleep-related worry and physiological aspects of anticipatory anxiety.

\section{Assessment points and intervention}

Baseline attentional bias assessment and questionnaire measures At baseline (day 1), after blind group allocation, all participants will complete an initial measure of sleep-related attentional bias using a dot-probe task consisting of the neutral and sleep-negative word pairs. The attentional bias assessment will be delivered online using Inquisit 3.0.3.2 software (Millisecond Software, Seattle, WA, USA).

The dot-probe assessment task will consist of 384 trials in total, with 10 repetitions of 48 word pairs delivered in randomised blocks (24 sleep-negative/neutral, and 24 neutral/neutral pairs at five repetitions each). In both the experimental and control condition, each trial starts with a fixation cross appearing in the centre of the screen for $500 \mathrm{~ms}$, followed by presentation of a word pair vertically aligned ( $3 \mathrm{~cm}$ separation), appearing for $500 \mathrm{~ms}$. After a gap of $100 \mathrm{~ms}$, a dot-probe (two small dots aligned either horizontally or vertically) appears either in the top or bottom position, remaining on the screen until a response key was pressed. Participants are specifically required to press the corresponding key, which indicated the position of the probe (key Z for left position; $\mathrm{M}$ for right position), as quickly and as accurately as possible. If participants responded incorrectly, error feedback will be provided triggering a $3 \mathrm{~s}$ delay. If a correct response is made, the screen will be cleared, and the next trial will commence after an interval of $500 \mathrm{~ms}$ (see Fig. 2). Here, for the initial and follow-up attentional bias assessments, the probes replace the negative and neutral member of each word pair with equal frequency to allow for the examination of vigilance and disengagement for sleep-negative stimuli.

The modified ABM version of the task will consist of 10 repetitions of 48 word pairs delivered in randomised blocks (all sleep-negative/neutral). Here, the probe always replaces the locus of the neutral word, promoting attentional avoidance of sleep-negative stimuli. In the non-ABM control condition, all task parameters are identical to the modified $A B M$ version, however the probes replaced the negative and neutral member of each word pair with equal frequency without promoting attention toward or away from negative words.

Following this, participants will complete: the ISI, SAMI, ASPQ, and SAAQ.

ABM intervention and control group procedure On days 3-7, using a computer, participants will be required to complete a dot-probe task prior to bed. The nature of the dot-probe task will depend on group allocation. Those in the experimental treatment condition will be provided with a modified version of the aforementioned dot-probe task such that the probe only appears in the location of the neutral word. Theoretically shifting (training) their attention away from the sleep-negative words. Whereas those in the control condition will complete the same task with probes replacing the negative and neutral member of each word pair with equal frequency. Following the task, participants will complete the Pre-Sleep Arousal Scale prior to bed. On awakening, participants will be required to complete an online version of the consensus sleep diary. 


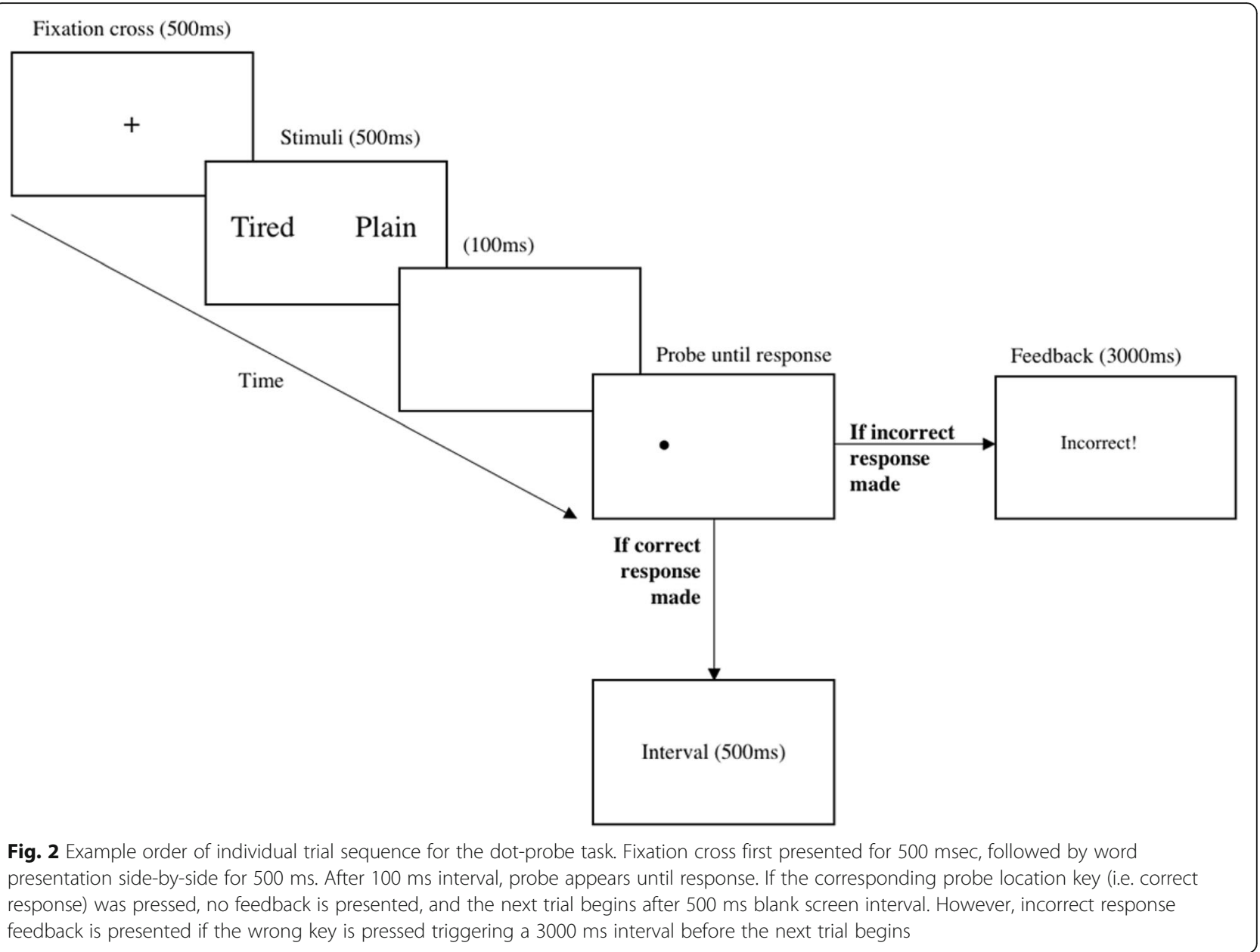

Post ABM assessment and questionnaire measures Following this 5-per week protocol, all participants will the complete the same attentional bias task (as completed at baseline) on the final day of the experimental phase (day 9). Moreover, the same questionnaire measures (ISI, SAMI, ASPQ, SAAQ) and sleep diary will also be completed.

Follow-up assessment At follow-up (day 16) participants will again complete the ISI, SAMI, ASPQ and SAAQ. On completion, participants will be provided with an Amazon e-gift voucher for taking part. Moreover, those in the control condition will be invited to take part in the experimental treatment if willing.

\section{Sample size rationale}

The current power estimation was based on the meta-analysis on ABM training for anxiety conducted by Hakamata and colleagues [27] who noted a mean effect size equal to Cohen's $d=.61$. In each group, we would require 44 participants based on a $t$ test, power of .80 and an alpha level of .05 (two-tailed).

\section{Statistical analyses}

Examination of accuracy and preparation of attentional bias data Only data from correct trials will be included in the final analysis. In addition, reaction times below $200 \mathrm{~ms}$, those above $3000 \mathrm{~ms}$, and those that are three standard deviations above each participant's individual mean will be eliminated [28].

Calculation of vigilance and disengagement To examine attentional bias, indices of vigilance and disengagement will be calculated. The vigilance index is calculated by subtracting the mean reaction time for negative stimuli from the mean reaction time for neutral stimuli: Vigilance index $=\mathrm{dN}, \mathrm{N}-\mathrm{dT}, \mathrm{N}$. Here, $\mathrm{dN}, \mathrm{N}$ represents dots replacing neutral words in the presence of another neutral word (i.e. neutral: neutral-neutral trials); and dT, $\mathrm{N}$ for dots replacing sleep-negative words in the presence of a neutral word (i.e. congruent: sleep-neutral trial). A positive score on the vigilance index indicates faster response to dots appearing after sleep-related as compared to neutral words [24], i.e. greater vigilance for 
'negative' stimuli. To calculate disengagement from sleep-related words, the mean reaction time for neutral trials are subtracted from the mean reaction time for trials where the dots replaced neutral stimuli in the presence of sleep-related stimuli: Disengagement index $=d N$, $\mathrm{T}-\mathrm{dN}, \mathrm{N}$. Here, $\mathrm{dN}, \mathrm{T}$ represents dots replacing neutral words in the presence of a sleep-related word (i.e. incongruent: sleep-neutral trials). A positive score on the disengagement index indicates slower responses to dots replacing neutral words in the presence sleep-related words [24], i.e. that attention was not directed to the neutral word but directed elsewhere (possibly the sleep-related word) when the sleep-related word is present. In sum, greater positive scores for both indices, vigilance and disengagement, are indicative of an attentional bias.

Analyses First, we will examine group differences in attentional bias indices at baseline with the expectation that there should be no group difference. Following this, we will examine differences in attentional bias indices at follow-up (i.e. day 9). To examine whether any attentional bias reduced amongst the experimental group, we will then examine the group (experimental versus control) $\times$ time (baseline versus post-ABM) interaction for each index of attentional bias (i.e. vigilance and disengagement). Second, to examine whether insomnia symptoms reduced amongst the experimental group, we will examine the group (experimental versus control) $\times$ time (baseline versus post-ABM versus follow-up) interaction. Third, to separately examine whether pre-sleep cognitive arousal and sleep-onset latency reduced amongst the experimental group on nights where the tasks were completed, we will examine the group (experimental versus control) $\times$ time (days 3 vs. 4 vs. 5 vs. 6 vs. 7 ) interactions. Finally, to explore the secondary aim we will employ a series of Pearson's correlational analyses to determine whether each of the attentional bias indices (i.e. vigilance and disengagement) are related to each subscale of the SAMI. Simple effects analyses will be performed to decompose any significant interactions as appropriate.

\section{Dissemination}

We will publish the results of this study in a peer-reviewed journal, regardless of the magnitude or direction of the results. Findings may also be presented at both national and international scientific meetings. The data will be made available online where possible, if permitted by journal policies.

Items in this protocol comply with the Standard Protocol Items: Recommendations for Interventional Trials (SPIRIT) Checklist (see Fig. 3 for the SPIRIT figure).

\section{Potential limitations}

It is relevant to note the procedural limitations that might arise during data collection. Considering the 16-day length of this protocol, we anticipate a degree of participant dropout. In addition, considering the online delivery of $\mathrm{ABM}$, some participants may experience technical difficulties in the absence of an experimenter.

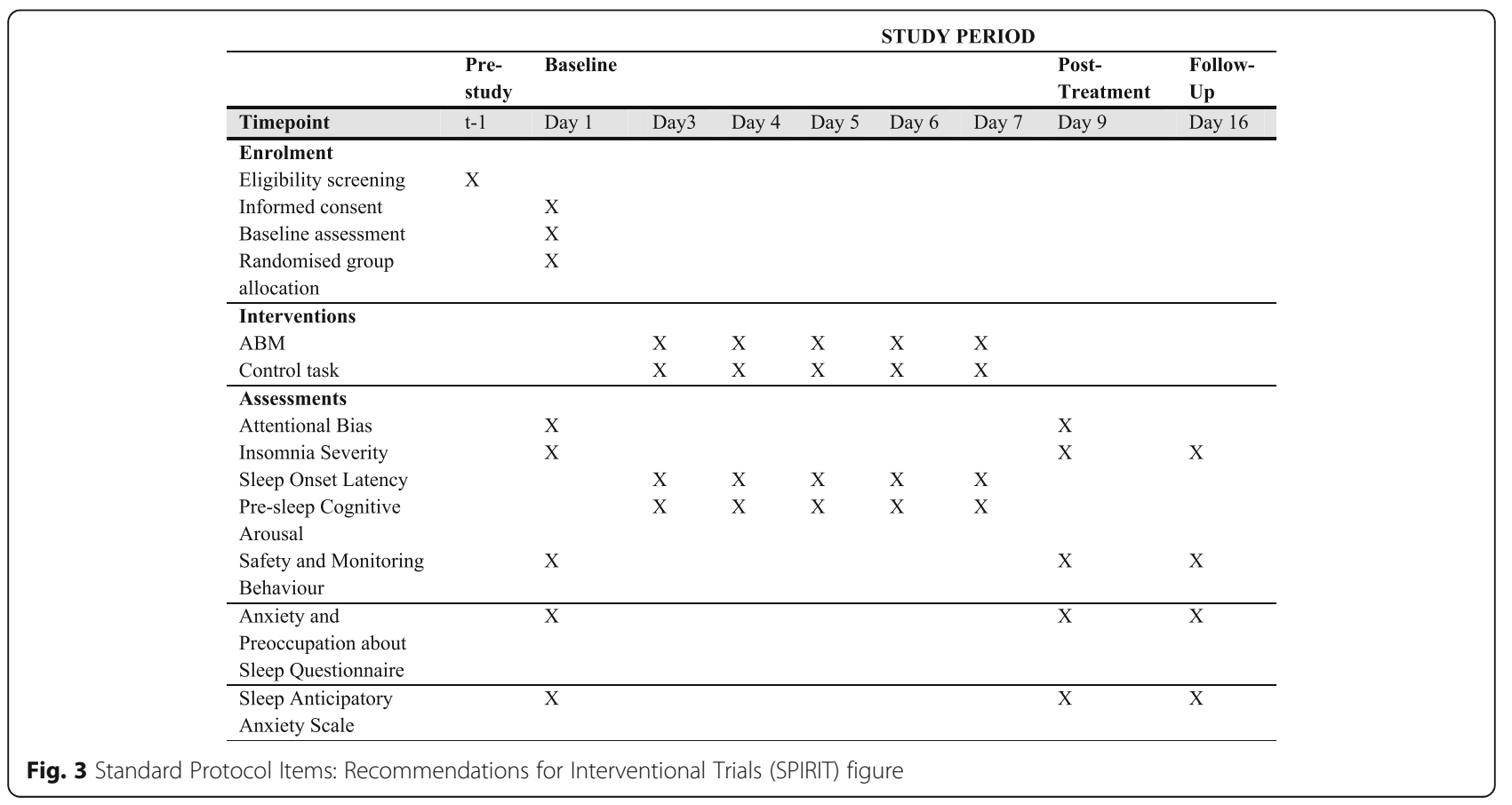




\section{Discussion}

Sleep-related attentional biases are considered to be a key factor in the development and maintenance of insomnia $[1,2]$. This study will determine whether attentional bias modification can be used to reduce the presence of an attentional bias, and whether this reduces symptom severity amongst individuals with insomnia.

\section{Trial status}

Recruitment of participants commenced on 10 May 2018 and is ongoing. Data collection will begin 1 November 2018.

\section{Abbreviations}

ABM: Attentional bias modification; APSQ: Anxiety and Preoccupation about Sleep Questionnaire; DSM-5: Diagnostic and statistical manual of mental disorders, $5^{\text {th }}$ edition; ISI: Insomnia Severity Index; PSAS: Pre-Sleep Arousal Scale; SAAS: Sleep Anticipatory Anxiety Scale; SAMI: Sleep Associated Monitoring Index; SOL: Sleep onset latency

\section{Funding}

This study is supported by the Sheffield Hallam University Department of Psychology, Sociology and Politics.

\section{Availability of data and materials}

The datasets analysed during the current study will be available from the corresponding author upon reasonable request.

\section{Authors' contributions}

$U A, N B, A Y$, and $L L$ are the chief investigators, conceived of the study, and contributed to the design. The experiment was programmed by $L N, B M$ and $J R$ contributed to the design. JS will coordinate the trial. UA, JS, AY, and LL will be responsible for the statistical analyses following data collection. UA drafted an initial version of the manuscript. Following input was received from all other authors. All authors approved the final manuscript.

\section{Ethics approval and consent to participate}

All participants are required to read the online information sheet and consent to participate in the study. This will include consent for their anonymised data to be published. The study has been granted ethics approval from the Sheffield Hallam University Research Ethics Committee (ER5451619) and the trial is registered at ISRCTN (ISRCTN11643569, registered on 5 June 2018)

\section{Consent for publication}

Not applicable.

\section{Competing Interests}

The authors declare that they have no competing interests.

\section{Publisher's Note}

Springer Nature remains neutral with regard to jurisdictional claims in published maps and institutional affiliations.

\footnotetext{
Author details

'Department of Psychology, Sociology and Politics, Sheffield Hallam University, Collegiate Crescent, Sheffield, South Yorkshire S10 2BP, UK. ${ }^{2}$ University of Western Australia, Elizabeth Rutherford Memorial Centre for the Advancement of Research on Emotion, Perth, Australia. ${ }^{3}$ Department of Psychology, The University of Sheffield, Sheffield, UK. ${ }^{4}$ Sleep and Circadian Neuroscience Institute, Nuffield Department of Clinical Neurosciences, Sir William Dunn School of Pathology, University of Oxford, Oxford, UK.
}

Received: 16 July 2018 Accepted: 25 September 2018

Published online: 19 October 2018

\section{References}

1. Espie CA, Broomfield NM, MacMahon KM, Macphee LM, Taylor LM. The attention-intention-effort pathway in the development of psychophysiologic insomnia: a theoretical review. Sleep Med Rev. 2006; 10(4):215-45.

2. Harvey AG. A cognitive model of insomnia. Behav Res Ther. 2002;40(8):869-93.

3. Akram U, Beattie L, Ypsilanti A, Reidy J, Robson A, Chapman AJ, Barclay NL. Sleep-related attentional bias for tired faces in insomnia: evidence from a dot-probe paradigm. Behav Res Ther. 2018;103:18-23.

4. Barclay NL, Ellis JG. Sleep-related attentional bias in poor versus good sleepers is independent of affective valence. J Sleep Res. 2013;22(4):414-21.

5. Jansson-Fröjmark M, Bermås M, Kjellén A. Attentional bias in insomnia: the dot-probe task with pictorial stimuli depicting daytime fatigue/malaise. Cogn Ther Res. 2013;37(3):534-46.

6. Lundh LG, Froding A, Gyllenhammar L, Broman JE, Hetta J. Cognitive bias and memory performance in patients with persistent insomnia. Cogn Behav Ther. 1997;26(1):27-35.

7. Macmahon KM, Broomfield NM, Espie CA. Attention bias for sleep-related stimuli in primary insomnia and delayed sleep phase syndrome using the dot-probe task. Sleep. 2006 Nov 1;29(11):1420-7.

8. Marchetti LM, Biello SM, Broomfield NM, MacMahon K, Espie CA. Who is pre-occupied with sleep? A comparison of attention bias in people with psychophysiological insomnia, delayed sleep phase syndrome and good sleepers using the induced change blindness paradigm. J Sleep Res. 2006; 15(2):212-21.

9. Spiegelhalder K, Espie C, Nissen C, Riemann D. Sleep-related attentional bias in patients with primary insomnia compared with sleep experts and healthy controls. J Sleep Res. 2008;17(2):191-6.

10. Akram U, Robson A, Ypsilanti A. Sleep-related attentional bias for faces depicting tiredness in insomnia: evidence from an eye-tracking study. J Clin Sleep Med. 2018;14(06):959-65

11. Beattie L, Bindemann M, Kyle SD, Biello SM. Attention to beds in natural scenes by observers with insomnia symptoms. Behav Res Ther. 2017;92:51-6.

12. Harris K, Spiegelhalder K, Espie CA, MacMahon KM, Woods HC, Kyle SD. Sleep-related attentional bias in insomnia: A state-of-the-science review. Clin Psychol Rev. 2015:42:16-27.

13. Woods HC, Scheepers C, Ross KA, Espie CA, Biello SM. What are you looking at? Moving toward an attentional timeline in insomnia: a novel semantic eye tracking study. Sleep. 2013;36(10):1491-9.

14. Hallion LS, Ruscio AM. A meta-analysis of the effect of cognitive bias modification on anxiety and depression. Psychol Bull. 2011;137(6):940.

15. Amir N, Weber G, Beard C, Bomyea J, Taylor CT. The effect of a singlesession attention modification program on response to a public-speaking challenge in socially anxious individuals. J Abnorm Psychol. 2008;117(4):860.

16. Milkins B, Notebaert L, MacLeod C, Clarke PJ. The potential benefits of targeted attentional bias modification on cognitive arousal and sleep quality in worryrelated sleep disturbance. Clin Psychol Sci. 2016;4(6):1015-27.

17. Clarke PJ, Bedford K, Notebaert L, Bucks RS, Rudaizky D, Milkins BC, MacLeod C. Assessing the therapeutic potential of targeted attentional bias modification for insomnia using smartphone delivery. Psychother Psychosom. 2016;85(3):187-9.

18. Lancee J, Yasiney SL, Brendel RS, Boffo M, Clarke PJ, Salemink E. Attentional bias modification training for insomnia: a double-blind placebo controlled randomized trial. PLoS One. 2017;12(4):e0174531.

19. American Psychiatric Association. Diagnostic and statistical manual of mental disorders $\left(\mathrm{DSM}-5^{\oplus}\right)$. American Psychiatric Pub; 2013.

20. Bastien $\mathrm{CH}$, Vallières $\mathrm{A}$, Morin CM. Validation of the Insomnia Severity Index as an outcome measure for insomnia research. Sleep Med. 2001 Jul 1;2(4): 297-307.

21. Spoormaker VI, Verbeek I, van den Bout J, Klip EC. Initial validation of the SLEEP-50 questionnaire. Behav Sleep Med. 2005;3(4):227-46.

22. Carney CE, Buysse DJ, Ancoli-Israel S, Edinger JD, Krystal AD, Lichstein KL, Morin CM. The consensus sleep diary: standardizing prospective sleep selfmonitoring. Sleep. 2012;35(2):287-302.

23. Nicassio PM, Mendlowitz DR, Fussell JJ, Petras L. The phenomenology of the pre-sleep state: the development of the pre-sleep arousal scale. Behav Res Ther. 1985;23(3):263-71. 
24. Semler CN, Harvey AG. Monitoring for sleep-related threat: a pilot study of the Sleep Associated Monitoring Index (SAMI). Psychosom Med. 2004;66(2): $242-50$.

25. Jansson-Fröjmark M, Harvey AG, Lundh LG, Norell-Clarke A, Linton SJ. Psychometric properties of an insomnia-specific measure of worry: the anxiety and preoccupation about sleep questionnaire. Cogn Behav Ther 2011;40(1):65-76.

26. KuoTF RM, Bootzin RR, Shoham S. Construct validity of the Sleep Anticipatory Anxiety Questionaire (SAAQ). Sleep Research. 1994;231(1):37-43.

27. Hakamata Y, Lissek S, Bar-Haim Y, Britton JC, Fox NA, Leibenluft E, Ernst M, Pine DS. Attention bias modification treatment: a meta-analysis toward the establishment of novel treatment for anxiety. Biol Psychiatry. 2010;68(11): 982-90

28. Salemink E, van den Hout MA, Kindt M. Selective attention and threat: quick orienting versus slow disengagement and two versions of the dot probe task. Behav Res Ther. 2007:45(3):607-15.

Ready to submit your research? Choose BMC and benefit from:

- fast, convenient online submission

- thorough peer review by experienced researchers in your field

- rapid publication on acceptance

- support for research data, including large and complex data types

- gold Open Access which fosters wider collaboration and increased citations

- maximum visibility for your research: over $100 \mathrm{M}$ website views per year

At $\mathrm{BMC}$, research is always in progress.

Learn more biomedcentral.com/submissions 